\title{
Anisotropic Nuclear Inelastic Scattering of an Iron(II) Molecular Crystal
}

\author{
H. Paulsen, ${ }^{1}$ R. Benda, ${ }^{1}$ C. Herta, ${ }^{1}$ V. Schünemann, ${ }^{1}$ A. I. Chumakov, ${ }^{2}$ L. Duelund, ${ }^{1}$ H. Winkler, ${ }^{1}$ \\ H. Toftlund, ${ }^{3}$ and A. X. Trautwein ${ }^{1}$ \\ ${ }^{1}$ Institut für Physik, Medizinische Universität Lübeck, D-23538 Lübeck, Germany \\ ${ }^{2}$ European Synchrotron Radiation Facility, Boîte Postale 220, F-38043 Grenoble, France \\ ${ }^{3}$ Department of Chemistry, University of Odense, DK-5230 Odense, Denmark
}

(Received 8 September 2000)

\begin{abstract}
Nuclear inelastic scattering (NIS) spectra were recorded for a monocrystal of the spin-crossover complex $\quad[\mathrm{Fe}($ tptMetame $)]\left(\mathrm{ClO}_{4}\right)_{2}$ (tptMetame $=1,1,1$-tris $\{[\mathrm{N}$-(2-pyridylmethyl)-N-methylamino $]$ methyl\}ethane) at $T=30 \mathrm{~K}$ (low-spin state) and at room temperature (high-spin state) for different crystal orientations. The high energy resolution $(0.65 \mathrm{meV})$ allowed us to resolve individual molecular vibrations which were unambiguously identified by density functional calculations. From the NIS spectra for the first time the angular-resolved iron-partial density of phonon states (PDOS) was extracted. The PDOS corroborates a vibrational entropy difference as driving force of the spin transition.
\end{abstract}

DOI: 10.1103/PhysRevLett.86.1351

Four decades ago, one year after Mössbauer discovered the recoilles absorption of gamma quanta by nuclei bound in crystals, Visscher pointed out how this new effect could be used to determine the frequency distribution of crystal lattice vibrations [1]. Only recently, when fast detectors and synchrotrons of the third generation became available, this vision could be realized leading to the method of nuclear inelastic scattering (NIS) of synchrotron radiation [2] which has become a valuable tool for the investigation of lattice dynamics in solids and of molecular dynamics in liquids and gases. The extremely narrow bandwidth of nuclear resonances of Mössbauer nuclei is used to create or annihilate phonons or to excite or deexcite molecular vibrations by detuning the energy of a photon from that of the nuclear resonance. This method has been applied to such different samples like the $\alpha$ and $\epsilon$ phases of iron at various temperatures and pressures, iron alloys and oxides, or biological substances like myoglobin $[3,4]$. NIS measurements have been performed on amorphous or polycrystalline samples, and anisotropic measurements on single crystals were done with ferric borate $[5,6]$ and guanidinium nitroprusside [7]. So far most NIS experiments have been performed with samples containing ${ }^{57} \mathrm{Fe}$, but recently also experiments on $\beta$-Sn (using ${ }^{119} \mathrm{Sn}$ ) have been reported [8].

In comparison to other spectroscopic techniques, which are used for studying lattice dynamics, e.g., inelastic neutron scattering, infrared or Raman spectroscopy, the nuclear inelastic scattering method has a special feature that makes it suitable for complementary investigations: the NIS method aims at the dynamics of the Mössbauer nucleus alone and allows one to extract only those normal modes from the complex vibrational spectrum of large metal complexes that are connected with a considerable mean-square displacement of the metal center. The measured anisotropic probability density $S(E, \hat{\boldsymbol{k}})$ at inverse temperature $\beta$, which depends on the difference $E$ between the photon energy and the nuclear resonance energy
PACS numbers: 76.80.+y, 31.15.Ew, 33.20.Tp, 33.25.+k

and on the normalized wave vector $\hat{\boldsymbol{k}}$ of the photon, can be expanded into a sum of multiphonon contributions

$$
\begin{aligned}
S(E, \hat{\boldsymbol{k}})= & f_{\mathrm{LM}}(\hat{\boldsymbol{k}}) \delta(0)+f_{\mathrm{LM}}(\hat{\boldsymbol{k}}) \sum_{n=1}^{\infty} S_{n}(E, \hat{\boldsymbol{k}}), \\
S_{1}(E, \hat{\boldsymbol{k}})= & g_{\mathrm{Fe}}(E, \hat{\boldsymbol{k}})\left(E_{R} / E\right)\left[1-e^{-\beta E}\right]^{-1}, \\
S_{n}(E, \hat{\boldsymbol{k}})= & \frac{1}{n} \int_{-\infty}^{+\infty} S_{1}\left(E^{\prime}, \hat{\boldsymbol{k}}\right) S_{n-1}\left(E-E^{\prime}, \hat{\boldsymbol{k}}\right) d E^{\prime} \\
& (n \geq 2),
\end{aligned}
$$

where the iron-partial density of states [6],

$$
g_{\mathrm{Fe}}(E, \hat{\boldsymbol{k}})=\sum_{j, \boldsymbol{q}} \delta\left[E-\hbar \omega_{j}(\boldsymbol{q})\right]\left|\hat{\boldsymbol{k}} \cdot \boldsymbol{e}_{j, \mathrm{Fe}}(\boldsymbol{q})\right|^{2},
$$

contains contributions of all phonons weighted by the projection of their polarization vectors $\boldsymbol{e}_{j, \mathrm{Fe}}$ for the resonant iron nucleus onto the normalized wave vector $\hat{\boldsymbol{k}}$ of the photon. Here $E_{R}=1.956 \mathrm{meV}$ is the recoil energy of a free ${ }^{57} \mathrm{Fe}$ nucleus and $\hbar \omega_{j}(\boldsymbol{q})$ is the energy of a phonon of branch $j$ with wave vector $\boldsymbol{q}$ and $f_{\mathrm{LM}}(\hat{\boldsymbol{k}})$ is the anisotropic Lamb-Mössbauer factor. $f_{\mathrm{LM}}(\hat{\boldsymbol{k}})$ describes the probability of elastic scattering and can be determined directly from the area below the elastic peak since $S(E, \hat{\boldsymbol{k}})$ is normalized to unity. In order to retrieve the iron-partial density of states (PDOS) from the experimental spectrum, the elastic peak $f_{\mathrm{LM}}(\hat{\boldsymbol{k}}) \delta(0)$ has to be subtracted from the measured $S(E, \hat{\boldsymbol{k}})$ and the multiphonon contributions have to be eliminated using the Fourier transformation [6]. From the remaining inelastic part, $f_{\mathrm{LM}}(\hat{\boldsymbol{k}}) S_{1}(E, \hat{\boldsymbol{k}})$, the anisotropic PDOS can be obtained by reordering Eq. (2). In case of molecular crystals, and if the interactions between interand intramolecular modes can be neglected, the molecular part of the anisotropic probability density can be obtained from electronic structure calculations leading to a more complex expression for $S(E, \hat{\boldsymbol{k}})$ [7]. In this approximation the molecular part of the PDOS can be formulated as 


$$
g_{\mathrm{Fe}}^{(\mathrm{mol})}=\sum_{j} \delta\left(E-\hbar \omega_{j}\right)\left(\hat{\boldsymbol{k}} \cdot \boldsymbol{b}_{j}\right)^{2},
$$

where $\omega_{j}$ are the vibrational frequencies of the molecule and $\boldsymbol{b}_{j}$ is the projection of the $j$ th eigenvector of the dynamical matrix into the three-dimensional subspace of the iron coordinates. The molecular quantity $\boldsymbol{b}_{j}$ corresponds to the phonon polarization vector $\boldsymbol{e}_{j, \mathrm{Fe}}(\boldsymbol{q})$ in the solid state. The molecular PDOS defined by Eq. (5) comprises only those contributions to the sum in Eq. (4) which are due to optical phonons, whereas the contributions from acoustical phonons are not included in Eq. (5).

With the help of the PDOS vibrational entropy differences in $\mathrm{Fe}_{3} \mathrm{Al}$ alloys with different local ordering have recently been investigated [3]. In the present study the PDOS is used to elucidate the influence of spin states on the vibrational entropy of a certain class of molecular crystals, the so-called spin-crossover complexes. These complexes exhibit a thermally driven change of the spin state of the metal center. For many of these complexes the transition from the low-spin (LS) state to the high-spin (HS) state and vice versa can also be induced by irradiation with light, and they are therefore promising materials for optical information storage and display devices [9]. The driving force for the thermally induced spin transition is the difference of entropy between the HS and the LS states, the major part of which arises from the decrease of vibrational frequencies that can be observed when passing from the LS to the HS state. The most prominent frequency shift can be attributed to the iron-ligand bond-stretching modes that are directly influenced by the change of the spin state. Exactly these modes give rise to the dominant inelastic peaks of NIS spectra of iron molecular crystals; this explains the value of the NIS method for the understanding of the spin crossover. First NIS measurements on spin-crossover complexes have been performed for $\left[\mathrm{Fe}(\mathrm{tpa})(\mathrm{NCS})_{2}\right][\mathrm{tpa}=$ tris(2-pyridylmethyl)amine] and for $\left[\mathrm{Fe}(\mathrm{bpp})_{2}\right][\mathrm{bpp}=$ 2,6-bis(pyrazol-3-yl)pyridine] with an energy resolution of 6 and $1.7 \mathrm{meV}$, respectively [10]. These measurements exhibit a shift of the average iron-ligand bond-stretching frequencies, but the low energy resolution has prohibited separation of single vibrational modes. With the recent improvement of high-resolution monochromators the experimental energy bandwidth could be lowered to $0.65 \mathrm{meV}$ [11], which allows one to resolve individual vibrational modes [12]. In the present study NIS measurements are presented for the first time which reveal the angular dependency of the vibrational modes of a molecular crystal. For this study the iron(II) spin-crossover complex $[\mathrm{Fe}($ tptMetame $)]\left(\mathrm{ClO}_{4}\right)_{2} \quad($ tptMetame $=1,1,1$ tris $\{[\mathrm{N}$-(2-pyridylmethyl)-N-methylamino $]$ methyl\}ethane) was selected because all molecules in the unit cell have the same orientation of their threefold symmetry axis, namely, parallel to the crystallographic $\boldsymbol{a}$-axis. This complex, which exhibits a complete spin transition at $228 \mathrm{~K}$, was prepared as described earlier [13] with $20 \%$ enrichment of ${ }^{57} \mathrm{Fe}$. Suitable crystals in the form of $2 \mathrm{~mm}$ long needles with about $0.2 \mathrm{~mm}$ thickness were obtained by recrystallization from a water acetonitrile (1:1) solution. An additional sample was prepared where about $1 \%$ of the $\mathrm{Fe}(\mathrm{II})$ ions have been replaced by $\mathrm{Mn}$ (II) ions in order to allow EPR measurements. X-ray diffraction measurements were performed at $120 \mathrm{~K}$ and at $260 \mathrm{~K}$ on the same single crystal that was used for the NIS experiment. The x-ray measurements confirmed that the sample has crystallized in the space group $P 2_{1} / n$ as reported earlier [13] and revealed that the needle axis of the sample coincides with the crystallographic $\boldsymbol{a}$-axis. EPR measurements on the $\mathrm{Mn}(\mathrm{II})$-doped crystal were performed for the same purpose at room temperature on a Bruker EMX 113 spectrometer operated at $X$ band and equipped with a Bruker $\mathrm{TM}_{110}$ high-sensitivity cavity. The crystal was mounted on its major faces and rotated with a Bruker ER 218 PG1 goniometer about the normal

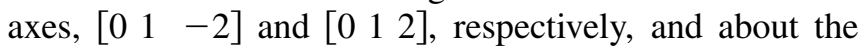
needle axis $\left[\begin{array}{lll}1 & 0 & 0\end{array}\right]$. The applied magnetic field was in all cases perpendicular to the rotation axis. The lack of angular variation in the EPR spectrum for the rotation about $\left[\begin{array}{lll}1 & 0 & 0\end{array}\right]$ confirmed the former assignment of crystal axes by $\mathrm{x}$-ray diffraction.

NIS spectra were recorded at the Nuclear Resonance Beamline ID 18 of the European Synchrotron Radiation Facility (ESRF) in Grenoble, France. The $6 \mathrm{GeV}$ electron storage ring was operated in 16 bunch mode with a purity of the filling better than $10^{-9}$. The incident beam was monochromatized by a $\operatorname{Si}(111)$ double-crystal premonochromator to a bandwidth of $2.5 \mathrm{eV}$. A further decrease of bandwidth down to $0.65 \mathrm{meV}$ was obtained with a refractive beryllium collimator and a high-resolution monochromator [11]. This beam was used to excite the $14.413 \mathrm{keV}$ nuclear level of ${ }^{57} \mathrm{Fe}$ in the sample. An avalanche photodiode with a time resolution of less than $1 \mathrm{~ns}$ has been used as detector [14]. The data were collected during several energy scans with 400 steps on average, each with $0.25 \mathrm{meV}$ stepsize and $10 \mathrm{~s}$ measuring time. The sample was mounted in a closed-cycle cryostat to permit measurements at different temperatures.

A first NIS spectrum of the LS isomer was recorded at $30 \mathrm{~K}$ with the crystallographic $\boldsymbol{a}$-axis parallel to the wave vector $\boldsymbol{k}$ of the incoming synchrotron radiation. A second and two further NIS spectra were recorded at $30 \mathrm{~K}$ with the $\boldsymbol{a}$-axis perpendicular to the wave vector $\boldsymbol{k}$ and parallel to the polarization axis of the synchrotron radiation. The third and the fourth spectra were recorded after rotating the crystal about $\boldsymbol{a}$ by $45^{\circ}$ each time. The latter three orientations yielded practically identical spectra, so that only the summation is shown (Fig. 1). After warming up the sample a spectrum of the HS isomer was recorded at room temperature (Fig. 1). At low temperature the LambMössbauer factor $f_{\mathrm{LM}}$ is close to one $\left(f_{\mathrm{LM}} \approx 0.88\right)$ and the NIS spectrum of the LS isomer is dominated by the 


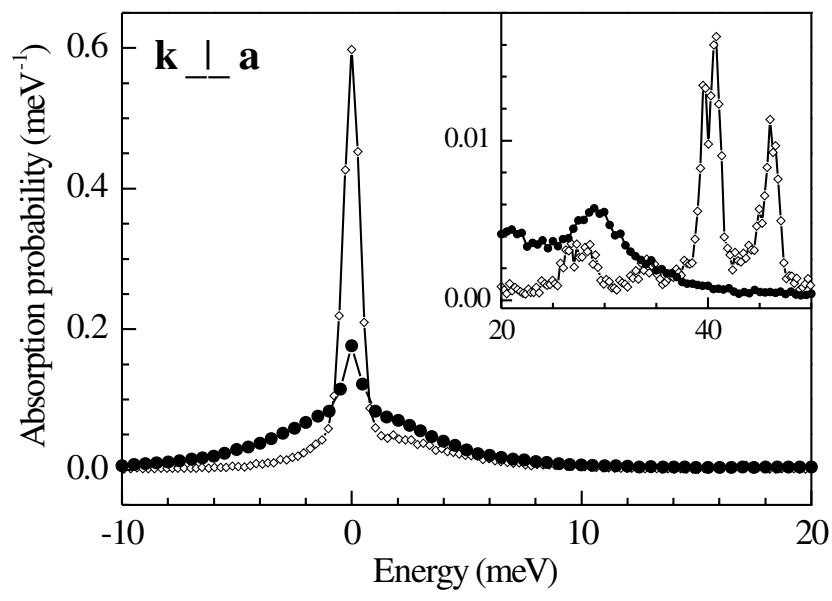

FIG. 1. NIS spectra measured at $T=30 \mathrm{~K}(\diamond)$ and at room temperature ( $)$ for $\boldsymbol{k} \perp \boldsymbol{a}$. The inset shows the energy range of the iron-ligand bond-stretching modes. The solid lines are guides to the eyes.

elastic peak. At room temperature $f_{\mathrm{LM}}$ decreases to 0.16 and only a small elastic peak is observed on top of a broad underground that is due to multiphonon creation and annihilation.

The PDOS of the LS isomer for $\boldsymbol{a} \perp \boldsymbol{k}$ [Fig. 2(a)], which was extracted from the measured probability density, exhibits two dominant peaks at 40 and $46 \mathrm{meV}$ as well as several smaller peaks in the range from 10 to $40 \mathrm{meV}$. With $\boldsymbol{a} \| \boldsymbol{k}$ [Fig. 2(b)] one dominant peak appears at $44 \mathrm{meV}$ accompanied by several smaller peaks with energies below $40 \mathrm{meV}$. The PDOS of the HS isomer for $\boldsymbol{k} \perp \boldsymbol{a}$ shows one dominant peak at $30 \mathrm{meV}$ and a smaller peak at $20 \mathrm{meV}$ (Fig. 3) both being broader than those observed for the LS isomer.

For the interpretation of the experimentally gained PDOS, density functional theory (DFT) calculations were performed for the HS and LS isomers of the $[\mathrm{Fe}(\text { tptMetame })]^{2+}$ cation in vacuo using the B3LYP method, implemented in the GAUSSIAN98 program system together with the Dunning-Huzinaga all electron double zeta basis set for $\mathrm{H}, \mathrm{C}$, and $\mathrm{N}$ and the Los Alamos effective core potential plus double zeta basis set on Fe [15]. The geometry of the cation was fully optimized and the frequencies $\omega_{j}$ and normal modes of molecular vibration were calculated. The calculated PDOS was folded with a normalized Gaussian with $1 \mathrm{meV}$ full linewidth.

Comparison of the experimental and the calculated PDOS for the HS and the LS isomer gives an overall agreement for the essential features with the exception of the acoustic contributions which are not included in the molecular model. The acoustic part, however, extends only up to $5 \mathrm{meV}$ and is roughly the same for the HS and the LS isomers. With the help of the calculated normal modes the two predominant peaks at 40 and $46 \mathrm{meV}$ for $\boldsymbol{k} \perp \boldsymbol{a}$ as well as the predominant peak at $44 \mathrm{meV}$ and the small peak at $41 \mathrm{meV}$ for $\boldsymbol{k} \| \boldsymbol{a}$ in the PDOS of the LS isomer can be assigned to iron-ligand bond-stretching

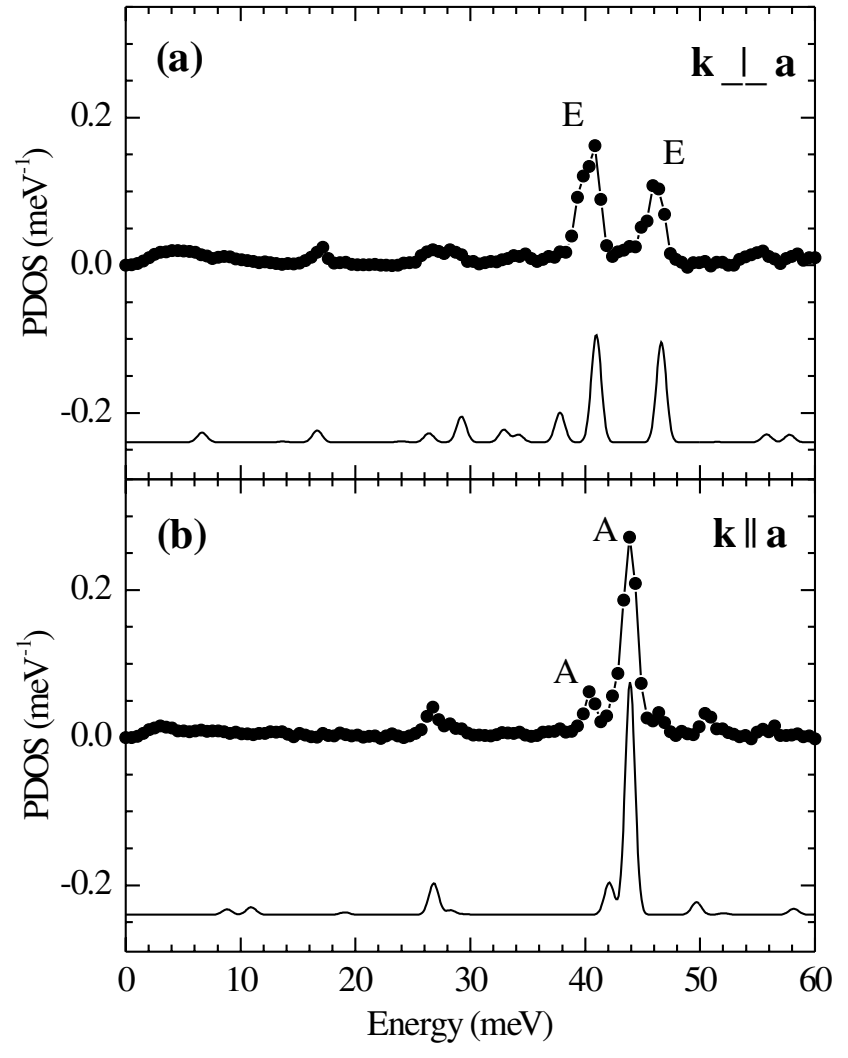

FIG. 2. Experimental PDOS (-) at $T=30 \mathrm{~K}$ for $\boldsymbol{k} \perp \boldsymbol{a}$ (a) and $\boldsymbol{k} \| \boldsymbol{a}$ (b). For better comparison the calculated PDOS (solid lines) is shifted and reduced in height by a factor of 2 .

modes. Considering an ideal $\left[\mathrm{FeN}_{6}\right]$ octahedron there exist six Fe-N bond-stretching modes which transform according to the $A_{1 g}, E_{g}$, and $T_{1 u}$ irreducible representations of the point group $O_{h}$. The gerade modes $A_{1 g}$ and $E_{g}$ are not connected with a displacement of the iron nucleus, and thus only the three $T_{1 u}$ bond-stretching modes can be detected by NIS. Actually, the first coordination sphere of the iron in the $[\mathrm{Fe}($ tptMetame $)]$ complex has

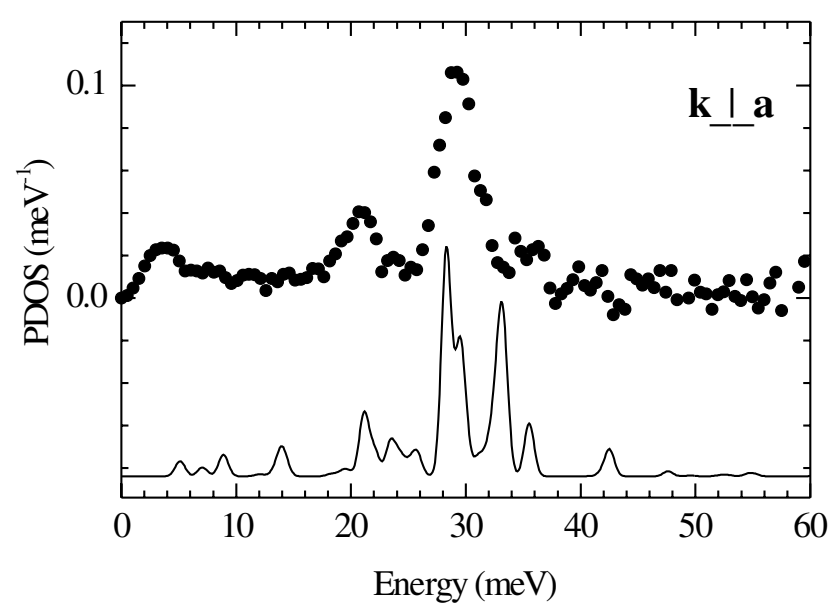

FIG. 3. Experimental PDOS (-) at room temperature. For better comparison the calculated PDOS (solid line) is shifted and reduced in height by a factor of 2 . 
to be regarded as a distorted $\left[\mathrm{FeN}_{6}\right]$ octahedron with $C_{3}$ symmetry. Because of the lower symmetry the vibrational $T_{1 u}$ term is split into an $A$ and a doubly degenerate $E$ term. In this case all six bond-stretching modes are visible by NIS: two pairs of doubly degenerate $E$ modes are connected with a displacement of the iron nucleus within the equatorial plane and are visible if $\boldsymbol{k}$ lies within this plane that is perpendicular to the threefold symmetry axis [Fig. 2(a)]. The remaining two $A$ modes are connected with a displacement of the iron nucleus along the molecular symmetry axis [Fig. 2(b)]. The smaller peak at $41 \mathrm{meV}$ corresponds to the nearly fully symmetric breathing mode where the iron nucleus is only slightly participating in the vibration.

The experimental PDOS for the HS isomer at room temperature with $\boldsymbol{k} \perp \boldsymbol{a}$ exhibits one dominant peak at about $30 \mathrm{meV}$, whereas the calculation yields two peaks at 28 and $33 \mathrm{meV}$ which correspond to iron-ligand bond-stretching modes. Apparently these modes are shifted by $10 \mathrm{meV}$ to lower energy when passing from the LS to the HS state. A detailed comparison of the calculated and the experimentally gained PDOS of the HS isomer at room temperature is not as straightforward as in the case of the LS isomer. The DFT calculations indicate a lowering of the molecular symmetry from $C_{3}$ to $C_{1}$ due to the Jahn-Teller effect upon crossover to the HS state. But at sufficiently high temperatures an averaged PDOS is measured, which can reflect a higher symmetry. This is a possible reason why the experimental PDOS exhibits only one broad peak at $30 \mathrm{meV}$.

The NIS spectra presented in this study allowed one for the first time to identify individual anisotropic normal modes of vibration of a molecular crystal. Remarkable agreement could be achieved between the experimental spectra and the spectra that were retrieved from density functional calculations. With the help of the PDOS the decrease of the vibrational entropy when passing from the HS to the LS state was visualized. Since the PDOS remains unchanged in the range of the acoustic phonons upon spin crossover the entropy decrease could be attributed solely to the optical phonons. Comparison of the experimentally obtained with the calculated PDOS revealed that the major contribution to the entropy difference arises from the ironligand bond-stretching vibrations.

The authors acknowledge the support by A. Hazel, R. Rüffer, and by the relevant ESRF services, and the financial support by the European Union (ERB-FMRX-CT0199) via the TMR-TOSS-network, by the German Research Foundation (DFG) and by the German Federal Ministry for Education, Science, Research and Technology (BMBF).
[1] W. M. Visscher, Ann. Phys. (N.Y.) 9, 194 (1960).

[2] M. Seto, Y. Yoda, S. Kikuta, X. W. Zhang, and M. Ando, Phys. Rev. Lett. 74, 3828 (1995); W. Sturhahn, T. S. Toellner, E. E. Alp, X. Zhang, M. Ando, Y. Yoda, S. Kikuta, M. Seto, C. W. Kimball, and B. Dabrowski, ibid. 74, 3832 (1995).

[3] B. Fultz, T. A. Stephens, W. Sturhahn, T. S. Toellner, and E. E. Alp, Phys. Rev. Lett. 80, 3304 (1998).

[4] R. Lübbers, H.F. Grünsteudel, A. I. Chumakov, and G. Wortmann, Science 287, 1250 (2000); K. Achterhold, C. Keppler, U. van Bürck, W. Potzel, P. Schindelmann, E.-W. Knapp, B. Melchers, A. I. Chumakov, A. Q. R. Baron, R. Rüffer, and F. Parak, Eur. Biophys. J. 25, 43 (1996); A. I. Chumakov, R. Rüffer, A. Q. R. Baron, H. Grünsteudel, and H.F. Grünsteudel, Phys. Rev. B 54, 9596 (1996).

[5] A. I. Chumakov, R. Rüffer, A. Q. R. Baron, H. Grünsteudel, H. F. Grünsteudel, and V. G. Kohn, Phys. Rev. B 56, 10758 (1997).

[6] V. G. Kohn, A. I. Chumakov, and R. Rüffer, Phys. Rev. B 58, 8437 (1998); V. G. Kohn and A. I. Chumakov, Hyperfine Interact. 125, 205 (2000).

[7] H. Paulsen, H. Winkler, A. X. Trautwein, H. Grünsteudel, V. Rusanov, and H. Toftlund, Phys. Rev. B 59, 975 (1999).

[8] A.I. Chumakov, A. Barla, R. Rüffer, J. Metge, H.F. Grünsteudel, H. Grünsteudel, J. Plessel, H. Winkelmann, and M. M. Abd-Elmeguid, Phys. Rev. B 58, 254 (1998); A. Barla, R. Rüffer, A. I. Chumakov, J. Metge, J. Plessel, and M.M. Abd-Elmeguid, ibid. 61, R14 881 (2000).

[9] P. Gütlich, H. Hauser, and H. Spiering, Angew. Chem., Int. Ed. Engl. 33, 2024 (1994); H. Toftlund, Coord. Chem. Rev. 94, 67 (1989).

[10] H. Grünsteudel, H. Paulsen, W. Meyer-Klaucke, H. Winkler, A. X. Trautwein, H. F. Grünsteudel, A. Q. Baron, A. I. Chumakov, R. Rüffer, and H. Toftlund, Hyperfine Interact. 113, 311 (1998); A. Chumakov and R. Rüffer, ibid. 113, 59 (1998).

[11] A. I. Chumakov, R. Rüffer, O. Leupold, A. Barla, H. Thiess, T. Asthalter, B. P. Doyle, A. Snigirev, and A. Q. R. Baron, Appl. Phys. Lett. 77, 31 (2000).

[12] A. Chumakov, R. Rüffer, O. Leupold, H. Grünsteudel, A. Barla, and T. Asthalter, ESRF Highlights-1999 (ESRF, Grenoble, 2000), p. 50.

[13] A. H. R. Al-Obaidi, K. B. Jensen, J. J. McGarvey, H. Toftlund, B. Jensen, S. E. J. Bell, and J. G. Carroll, Inorg. Chem. 35, 5055 (1996).

[14] A. Q. R. Baron, Nucl. Instrum. Methods Phys. Res., Sect. A 352, 665 (1995).

[15] M.J. Frisch et al., GAUSSIAN98, Revision A.7, Gaussian, Inc., Pittsburgh, PA, 1998. 